\title{
Barriers and facilitators to an outreach rehabilitation program delivered in nursing homes after hip fracture surgical repair
}

\author{
Donna M. Wilson ${ }^{1 *}$, Sandra L. Robertson ${ }^{1}$, C. Allyson Jones ${ }^{2}$, D. W. C. Johnston ${ }^{3}$, \\ Lauren A. Beaupre ${ }^{2}$ \\ ${ }^{1}$ Faculty of Nursing, University of Alberta, Edmonton, Canada; ${ }^{*}$ Corresponding Author: donna.wilson@ualberta.ca \\ ${ }^{2}$ Department of Physical Therapy, Faculty of Rehabilitative Medicine, University of Alberta, Edmonton, Canada \\ ${ }^{3}$ Department of Surgery, Faculty of Medicine and Dentistry, University of Alberta, Edmonton, Canada
}

Received 9 December 2012; revised 8 January 2013; accepted 15 January 2013

\section{ABSTRACT}

Objective: To identify and understand facilitators and barriers to implementing an Outreach rehabilitation program designed to improve postoperative recovery following hip fracture in longterm care residents. Residents of nursing home facilities are at considerable risk of hip fracture and minimal recovery following a hip fracture. Methods: Data were gathered over June-August, 2012 through semi-structured interviews or focus groups. Fifteen persons $(n=15)$ who were members of the Outreach rehabilitation team $(n=$ 8) or relevant nursing home staff $(n=7)$ were interviewed. Data analysis was guided by principles of grounded theory method. Findings: Three major themes that contributed to or hindered the Outreach rehabilitation program emerged, namely, 1) the division, the separate operation and delivery of rehabilitation services; 2) building bridges, or negotiating ways to communicate and work together; and 3) strength in the structure, the acceptance of the program and the perceived benefits of the program. One main challenge to program implementation concerned coordinating additional rehabilitation with the rehabilitation provided within the nursing homes. Facility staff was largely unaware of the program and were unprepared to work with Outreach team members. As the program progressed, the facility staff and Outreach team were able to collaborate to overcome resident health issues impeding recovery such as cognitive impairment, language barriers and post-surgical pain control needs. Facilitators included the consistency of Outreach team members and accessible facility staff, which contributed to effective communication and trust between the
Outreach team and facility staff. Facilitators also included support for the program by the Outreach team and facility staff, as well as the potential benefits of improved mobility and functional status among some program recipients. Conclusion: Although planning, implementation, and delivery of an Outreach rehabilitation program present some challenges, this study suggests that it is possible to deliver rehabilitation to older residents who fracture their hips in nursing homes.

Keywords: Hip Fracture; Rehabilitation; Qualitative Research; Nursing Homes; Continuing Care

\section{INTRODUCTION}

Hip fractures are unfortunately common among residents of nursing homes [1]. Nursing home residents are twice as likely to suffer a hip fracture as older community-dwelling persons [2]. This rate is not surprising, as the risk of hip fractures increases with age [3] and with disability [4]. Nursing home residents are often very old [5], and typically disabled and thus dependent on others [4]. Previous studies have shown residents of nursing homes experience lower recovery after hip fracture surgery than their community-dwelling counterparts [6]. One study revealed only $22 \%$ of nursing home residents recovered to their pre-fracture functional levels as compared to $71 \%$ of their community-dwelling counterparts [7].

Rehabilitation after hip fracture surgery is heavily dependent upon effective rehabilitation, yet rehabilitative services are highly variable across nursing home facilities, with many having limited in-house services [8]. Indeed, some would argue that rehabilitation is not a primary function of nursing homes given the residents' often advanced or end-stage ill health. Although demen- 
tia affects the majority of nursing home residents [4], a recent systematic review indicated that functional recovery after hip fracture surgery was similar across subjects who did and did not have dementia [9]. Given the known limitations in nursing home rehabilitative services, an Outreach rehabilitation program was designed and implemented to improve access to rehabilitation services after hip fracture for residents of nursing homes in Edmonton, Canada. A qualitative research study was carried out to identify and understand the barriers and facilitators to initiating this Outreach program.

\section{METHODS}

\subsection{Background}

Following ethics approval from a University of Alberta Research Ethics Board, nine nursing homes in Edmonton Alberta were approached for approval to have data collected for this study. All of these had residents receiving the rehabilitation provided by the Outreach team in addition to their own in-house rehabilitative services. Five of the nine facilities agreed to participate.

The Outreach team consisted of a Coordinator, Physical Therapists and Physical Therapy Assistants who provided 45 minutes rehabilitation sessions three times a week for 10 weeks to nursing home residents after the return to their nursing home following hip fracture surgery. People are typically discharged from hospital five days after hip fracture surgery, as long as they are medically stable. Before discharge, hospital patients in the city of Edmonton Alberta are treated through a regional care pathway that includes mobilization on the first postsurgery day and then daily rehabilitative care that emphasizes walking (unless contraindicated). The intent of the Outreach rehabilitation program was to augment the rehabilitation that residents would normally receive from the nursing home nurses and rehabilitation staff.

All nursing home residents who had been ambulatory prior to their hip fracture were eligible for participation in the Outreach program. The additional rehabilitation provided through the Outreach program was functionally directed at improved in-bed mobility, transfers from bed to chair and vice versa, walking within and outside their room, and improved balance; all of which are most commonly reported as having beneficial effects on function and mobility, and reduced fall risk [10]. Whenever a resident returned to their nursing home after hip fracture surgery, the Outreach Coordinator would contact both the nursing home administrator and rehabilitation staff within the facility to set up a mutually convenient time when the Outreach team and facility rehabilitation personnel could meet to assess the nursing home resident and plan coordinated services.

\subsection{Study Design}

An exploratory descriptive qualitative research study was conducted to identify and understand both the barriers and facilitators to the Outreach rehabilitation program. This study uses the principles of grounded theory, which include: 1) interviews of key participants; 2) an inductive generation of theory from the data as it were gathered; 3 ) an iterative or ongoing process of data collection and constant-comparative data analysis; and 4) the identification of and then connection of concepts to devise a theoretical framework consisting of themes and sub-themes [11]. The Glaserian grounded theory approach was specifically used, as it allows for flexibility, unpredictability, and creativity of theory generation from the data $[11,12]$. The grounded theory method was chosen as it permits the exploration and analysis of multiple data sources [12], allows multiple participant voices, and is acceptable for diverse perspectives and backgrounds [11]. However, as informed consent could not be obtained from most nursing home residents, only the Outreach team members and directly affected nursing home staff could be interviewed. A purposeful stratified sampling strategy was used to ensure representation from the Outreach team and nursing home staff.

\subsection{Participants}

Seven nursing home staff volunteered for the study, and each was interviewed individually. Three were Physical Therapists, two were Health Care Aides, and two were Registered Nurses. Eight Outreach team members were also interviewed individually or in focus groups. One participant was the Coordinator for the Outreach program, while the remaining participants were Physical Therapy Aides $(n=5)$ and Physical Therapists $(n=2)$. A total of fifteen $(n=15)$ participants were thus interviewed. All participants were female except one.

The nursing home facility participants had worked 10 years on average, with the range of experience 1.5 years to 32 years. Their average work hours were 0.63 FTE (full-time equivalent), or three days a week, with a 0.54 FTE to 1.0 FTE range. The Outreach team participants were hired on a casual basis, as their workload could not be predicted.

\subsection{Data Collection}

In grounded theory research, data are collected until saturation, a point when no new information or codes emerge [13]. Saturation was reached with the fifteen participants, a number that is comparable with previous qualitative studies exploring rehabilitation after hip fracture $[14,15]$. Throughout this study, after participants were recruited and voluntarily agreed to participate, interviews were conducted either as face-to-face individual 
interviews or in small focus groups. Focus groups have the advantage of gaining additional insights as participants can reflect on what other participants say [16]. Data were collected f From June to mid-August 2012, a $\$ 25$ gift certificate was provided to all participants to thank them for their participation. The individual interviews ranged in length from 11 to 37 minutes. The two focus group sessions lasted 37 and 48 minutes. Three primary questions were asked every time, with related follow-up questions to elicit further responses: 1) What factors or characteristics facilitated the Outreach program in the facility; 2) What factors or characteristics hindered the Outreach program in the facility? and 3)What changes would improve the implementation and adherence to an Outreach program?

All interviews and focus groups were tape recorded, with this work undertaken by a nurse researcher who received training in a classroom and on-site from a more experienced nurse researcher. Field notes were taken by the nurse researcher to record additional key information, assist with data analysis and thematic coding, and contribute to an audit trail for study rigor. The field notes were written as soon as possible after each interview and focus group, and these contained information on participant background demographics, notes from and about the interview, and the researcher's reflections. The audio tapes were transcribed by an independent transcriber, with two members of the research team reviewing and cleaning the transcripts prior to data analysis.

\subsection{Data Analysis}

Data analysis was conducted in keeping with the four recommended stages of grounded theory methods: 1) comparing data and coding for a category; 2) integrating the categories; 3) delimiting and saturation of the categories; and, 4) writing theory [12]. Data collection and constant comparison analysis continued until the data showed sufficient comprehensive information as well as no new emerging information therefore reaching theoretical saturation associated with grounded theory method [11]. As this study was small, and not intended to produce a robust theory, only descriptive themes were thus identified from the data.

The initial analysis was done individually with one experienced researcher using traditional paper methods and the other researcher using a computer-aided qualitative data analysis software program (ATLAS.ti). Transcripts, audio recordings and field notes were imported into the ATLAS.ti (version 7) program. Congruent with grounded theory methodology, data analysis started with the first transcript and continued concurrently with each additional transcript; each transcript was individually coded as well as compared to the previous transcript for emerging themes [12]. Initial open coding was done by assigning codes to relevant data segments. Throughout the data analysis, the groundedness of the code was evident in how many times the respective code was identified in the transcripts [17]. After this initial open coding by the two researchers working independently of one another, codes were grouped into families or categories of similar codes by the two researchers working together. Field notes and memos were used to further the data analysis from categories into emerging themes. As the two researchers grouped the data, differences between the researchers were noted and common sub-themes and themes were developed through ongoing discussions between the two primary researchers.

\section{FINDINGS}

Three themes emerged to highlight barriers and facilitators to implementing the Outreach rehabilitation program in nursing homes for residents following hip fracture surgery: 1) the division, or the separate operations and delivery of the two distinct rehabilitation services; 2) building bridges, or negotiating ways to communicate and work together; and 3) strength in the structure, or the acceptance of the program which was based in part on the perceived benefits of the program. Each is described below, with select quotes and other findings provided to illustrate and explain each theme.

\subsection{The Division}

The Outreach rehabilitation team and facility staff operated primarily as separate teams. This division contributed to challenges: 1) a lack of understanding about the Outreach program by the nursing home staff; 2) difficulties in coordinating the additional rehabilitation services with the usual in-house rehabilitation sessions; and 3) reduced Outreach team member access to resident information and on-site rehabilitation equipment. However, this division was only mainly present at the beginning of the involvement of Outreach team members in each facility.

The Outreach rehabilitation program was intended to complement the existing rehabilitation care in the nursing homes, thereby providing additional rehabilitation sessions during a phase in which most functional recovery occurs [18]. There was inconsistency in the rehabilitation offered to residents across the facilities. Although the frequency of rehabilitation services was based on the assessment of individual residents, facility policies often conflicted with the addition of external rehabilitation services. Some residents received daily rehabilitation sessions including weekends, while others only received the three rehabilitation sessions a week by the Outreach team. Once this was identified as an issue, it was corrected. Facility policies, overworked facility staff, and a 
lack of understanding in the facility about the program contributed to frustration among the Outreach and facility teams. One nursing home staff member stated:

They (patients) come back (from hospital) and our staffing is limited, so a program like this, even though we can't work together, three times a week and longer sessions each, is better than what we can provide. But as I said, it's hard to coordinate, I was not allowed to work together with the Outreach program staff, but I was able to just give information and monitor how they were doing.

The division of the two teams and limited interaction between them contributed to a lack of understanding about the Outreach program as a whole by the nursing home staff. An Outreach team member noted:

I think that if we could work together more, then they (the facility staff) would understand that they're still doing their part and we're doing our part... instead of being completely separate.

Another noted:

I didn't know they had an outbreak (of a serious contagious virus) and I went there to work, and the front desk said we were not supposed to come in.

Scheduling and timing conflicts were also present. The Outreach team and facility staff tried to accommodate each other by scheduling the days and time of the rehabilitation sessions, but the Outreach team often found the residents were not ready or available for the additional rehabilitation sessions. Scheduling conflicts were mainly found around meal times, church services, and recreational activities. Outreach team members commented:

We're waiting there for an hour; you know, waiting till the [church] service is done and then by the time the service is done, the patient is tired and not wanting to have rehabilitation.

Yeah, a lot of it was timing; like if we came after lunch there was no chance you would ever get someone after lunch, because they'd always wanted to sleep. But if you went in the morning usually it was pretty standard that you could get someone to be willing to do their exercises with you.

The timing of resident medications often conflicted with the Outreach team member's ability to perform rehabilitation sessions. Some residents were in pain or too drowsy from pain medications to participate. Outreach team members noted:

A couple times we had issues with the timing of medications. They would get pain medication right before we came, which would either be really helpful, or it would make them drowsy and they just didn't want to do anything at all. If there was a client who demonstrated behavior issues, or significant pain issues, then we would [be] ...dealing with medications that basically made them drowsy or knocked them out; and it was very, very hard to optimize interventions when they are under that much medication. So that was difficult, and I think trying to tweak the medications was an issue as far as trying to find the facility staff person who could speak for what was happening. The one thing that's very hard to control was, medications and trying to have the resident physically, mentally and behaviorally ready for optimal intervention.

The Outreach team also reported other difficulties, including access to residents' health care information, and up-to-date information on the residents' health status. Limited and busy facility staff made it difficult for the Outreach team members to locate the right facility staff member to gain information or address resident issues. Facility equipment and parking were not always available as well. Outreach team members reported:

Sometimes if no one was at the front desk, we would wait until someone can come, or grab someone but they might said—oh I don't the key, we have to get someone else to open the [resident's chart room] door.

There was one client early on that the physiotherapist that I was working with wanted her on the parallel bars, and we just weren't able to facilitate that because the nursing home staff were running their own physio programs and were using them. And so yeah, I guess equipment came into play there and that was a barrier.

In conclusion, the division or the separate operation and delivery of services by the nursing home staff and the Outreach team contributed to barriers of coordinating and complementing care, as well as difficulties with access to resident information and equipment. The implementation of the program produced challenges that highlighted the division of services between the Outreach and nursing home teams.

\subsection{Building Bridges}

After the Outreach rehabilitation program was initiated and it progressed within the nursing homes, efforts to build bridges, or the negotiating of ways to communicate and work together occurred. The proposed intention of the Outreach program was to optimize the recovery of each resident during the sub-acute phase of recovery after hip fracture. This intent was well-received by the nursing home staff. Both the Outreach team members and nursing home staff understood the potential benefits of the program and this outcome helped their efforts to try to work together in addressing the encountered barriers.

Three main facilitators for the collaboration of the teams were identified: 1 ) the communication binder that was used by Outreach team staff and nursing home staff; 2) working together on individual resident barriers for rehabilitation sessions, such as their cognitive and language barriers; and 3) having consistent nursing home 
staff and Outreach team members for each individual resident.

One major way of facilitating communication between the Outreach Rehabilitation team and the nursing home staff was a communication binder in which the Outreach program staff included specific information regarding the rehabilitation treatment plan. The resident's current health status, physiotherapist assessments, and rehabilitation session updates were written in the communication binder by both the nursing home staff and Outreach team members. This facilitated a clear connection between all persons involved in a resident's care, as illustrated by these quotes:

...it (communication binder) was available to the nurses and anybody. So it was a good communication tool actually.

I would leave them to do their thing, and the team would come, and you know, if they had questions or whatever, and you know we could communicate. I tried, I was not that great, but I did try to document everything, in the binder.

I think those binders were a huge help, the binders were already onsite, and so they knew that we were there for a purpose, we weren't just coming in and trying to do our own thing.

The Outreach team and nursing home staff found they needed to work together when addressing resident challenges; such as cognitive impairments, language barriers, behavioural issues, and increased risk for recurrent falls. The residents presented with complex health issues that required common planning and team work. Another facilitator was that the nursing home rehabilitation staff appreciated the extra help, especially on the weekends, as illustrated by these quotes:

I met with the physiotherapist who was assigned to this program, and we had a discussion about the resident and what we can achieve together.

When you have people with dementia, you can't necessarily do a class, you need to have one on one care, and when you do not have enough staff for the amount of people, it limits what you can do.

We do have a regular schedule here for the residents for twice and three times a week and our (physio) groups. So technically doing a resident every day is a lot of time that you are committing. It is not just the walking, you have bed exercises, language (barriers) ...you are looking at keeping this 45 minutes aside every day. So that is a challenge. I think it was a great help to have the other team here for the rest of the days.

Having the same Outreach team members going into their assigned nursing home also contributed to the building of trust about Outreach team members. The Outreach team members also appreciated having the same nursing home staff as partners when working with their residents, as they came to know each other's skills and abilities. However, consistency in both the Outreach team and facility rehabilitation staff was a challenge as both experienced limited availability and frequent staff turnover. Outreach team participants noted:

I think that was a huge piece too, these people are older and they, they thrive on routine, and so when you do have the same people coming in and pushing them beyond what they think they can do, it really benefits that patient.

I've been partnered up with some great people. I do like the consistency of the partner; so again, it's my preference to see the same person throughout the journey, if I can. And I think it's quite positive if I'm with them, the same PTA, and able to work with them throughout the process, as well.

Through these facilitators, bridges were built over time to negotiate ways to communicate and work together with everyone involved with the Outreach program. These strengths further contributed to the Outreach program and its' intended outcomes.

\subsection{Strength in the Structure}

Participants perceived that there were benefits of the program as it was structured. These benefits included not only some perceived physical improvements from select residents, but also nursing home staff were energized and motivated by the Outreach team's presence and collaborative work.

The facility rehabilitation staff indicated that the Outreach rehabilitation program comprised an enhancement of the usual facility rehabilitation services. Specifically, they noted that the Outreach team members were able to provide one-on-one sessions with residents, spend more time with the residents than what they had available, and that some residents appeared to benefit from the additional rehabilitation sessions each week. All of the Outreach team members and the nursing home staff who were interviewed observed potential improvements among some residents such as increased walking, improved bed mobility, and increasing independence in daily activities. In addition, some nursing home residents were described as being happy, grateful, and motivated for the extra rehabilitation sessions. These residents and families appeared to appreciate the focused attention, as well as the more regular consistency in rehabilitation care. A nursing home staff member and an Outreach team member illustrate these benefits:

We don't have time to keep coming back and encourage him, but I saw the Outreach team there; they can take time and they can give him time to rest, and do more and as much as he can. So I think that was big difference from us and the team members.

I think it's great, getting that extra physio is always 
good... I know some long term care facilities where it can only be done once or maximum twice, so it's great (to have more rehabilitation).

A lot of people, staff as well as families were quite grateful that we were coming in and being able to attend to their resident.

Many of the nursing home staff indicated they would like the Outreach program to continue and that it should expand into other rehabilitation services, such as post pelvic or rib fractures, and help residents with chronic health conditions such as multiple sclerosis (MS) and amyotrophic lateral sclerosis (ALS). The program was viewed as a success even if the resident failed to return to their previous functional status prior to the hip fracture.

Another structural benefit was that the Outreach team appeared to motivate the facility staff. Some facility staff admitted to learning and being energized by the Outreach team person coming into their facility. It was also helpful that the Outreach program did not increase their workload. Some nursing home staff participants noted:

It was extra, a kind of treat; their work reminded me, I think we should do our bed mobility... so it's a good reminder of a couple more things we should be doing.

So when staff find out you're going to make these patients nice and limber, and strong; getting them dressed or getting them to the bathroom, and doing transfers, it makes it so much easier for them.

As such, all research participants identified emerging benefits of the program. These benefits were oriented to the residents as well as the nursing home staff.

\section{DISCUSSION AND IMPLICATIONS OF FINDINGS}

This qualitative study was helpful for revealing barriers and facilitators to a newly implemented Outreach rehabilitation program for nursing home residents recovering after hip fracture surgery. As indicated, three central themes emerged: 1) the division, or the separate operation and delivery of services; 2) building bridges, negotiating ways to communicate and close the division between the two teams; and 3) strength in the structure, which outlines the emerging benefits and acceptance of the Outreach program. Across these themes, communication was critical to all persons involved with the Outreach program. Specifically, communication was critical to its introduction, as well as the ongoing work and emerging effectiveness of the program. Understandably, new programs may not be understood by the persons who may be impacted by them. Although the communication binder was a good resource for information, written information did not relieve the need for one-on-one communication from nursing home managers to staff about the program, and one-on-one communication between the Outreach team members and nursing home staff.

Another critical aspect was the perceived potential benefits of the program for nursing home residents, which was a major reason for the motivation of individuals on both teams to work together and support each other. Although the family member perspective was not gained through this study, both the Outreach team members and facility staff indicated that their approval of the program was based in part on families indicating that they were happy with the added rehabilitation services that were provided to their family member.

The structure of this program presented challenges for communication between and among the two teams, and for coordination of services. Having an additional offservice rehabilitation program and additional rehabilitation personnel providing care within the nursing home could be highly challenging. However, it should be noted that outsiders who work within a facility are considered boundary spanners, and research indicates that they are often highly influential as a result of this unique position [19].

A quantitative study is currently underway to measure the impact of the Outreach program on nursing home residents' recovery. Together, that quantitative study's findings and this qualitative study's findings may influence and encourage the initiation of the Outreach rehabilitation program in other nursing home facilities. These same findings may also encourage other jurisdictions to adopt an Outreach rehabilitation program approach to augment rehabilitation in nursing homes. If this occurs, the following recommendations should be of assistance:

- Ensure that Outreach team members and nursing home staff are aware of each other's roles and work, to reduce or prevent conflicts with scheduling and ensure resident access to rehabilitation sessions (either 5 times or 7 times each week for 10 weeks);

- Ensure clear and open communication lines are developed and maintained between the nursing home staff and the Outreach team members, such as through initial meetings with key persons and using a coordinator to facilitate ongoing communication;

- Develop a communication binder as face-to-face communication can be difficult to achieve and then encourage both nursing home staff and Outreach team members to use the binder;

- Assign Outreach staff to only select residents and select nursing homes, to facilitate effective and consistent communication between them and the nursing home staff.

\section{LIMITATIONS}

The small sample size and small number of focus groups may have limited data collection on facilitators and barriers to implementing the Outreach program. 
However, this may not be a major factor, as data saturation was reached. Another limitation was the minimal participation by nursing home managers, and the lack of involvement of nursing home residents and their families. Despite these limitations, our findings highlight some potential challenges and strengths to implementing an Outreach rehabilitation program in nursing homes. These findings will hopefully encourage similar pilot projects to increase rehabilitation access for persons recovering from hip fracture surgery in nursing homes.

\section{CONCLUSION}

Although the planning, implementation, and ongoing delivery of an Outreach Rehabilitation program for nursing home residents recovering from hip fracture surgery present some challenges, this study indicated that not only is a complementary rehabilitation program possible, but it appears to have some direct and indirect benefits for residents as well as the nursing home staff. This study was thus helpful for identifying barriers to implementing and delivering an Outreach rehabilitation program in piloted nursing homes in Edmonton Alberta, and for gaining insight into the potential benefits of such a program. The unique structure of the program, which introduced boundary spanners into the nursing homes, may have been a key feature for the relative ease by which this program was initiated and came to function in the involved nursing homes.

\section{ACKNOWLEDGEMENTS}

We would like to acknowledge the other members of the recovery after Hip Fracture in Geriatric Ambulatory Patients Living in Nursing Homes (REGAIN) team-Drs. S. R. Majumdar and J. Magaziner. Drs. Beaupre and Jones receive salary support from Alberta InnovatesHealth Solutions (formerly Alberta Heritage Foundation for Medical Research (AHFMR)) as Population Health Investigators. Research Funding Source: Alberta Innovates-Health Solutions.

\section{REFERENCES}

[1] Brennan, J., Johansen, A., Butler, J., Stone, M., Richmond, P., Jones, S., et al. (2003) Place of residence and risk of fracture in older people: A population-based study of over 65-year-old in cardiff. Osteoporosis International, 14, 515519. doi:10.1007/s00198-003-1404-5

[2] Norton, R., Campbell A.J., Reid, I.R., Butler, M., Currie, R., Robinson, E., et al. (1999) Residential status and risk of hip fracture. Age and Ageing, 28, 135-139. doi:10.1093/ageing/28.2.135

[3] Ronald, L., McGregor, M., McGrail, K., Tate, R. and Broemling, A. (2008) Hospitalization rates of nursing home residents and community-dwelling seniors in british columbia. Canadian Journal of Aging, 27, 109-115. doi:10.3138/cja.27.1.109
[4] Hirdes, J., Mitchell, L., Maxwell, C. and White, N. (2011) Beyond the "iron lungs of gerontology": Using evidence to shape the future of nursing homes in Canada. Canadian Journal on Aging, 30, 371-390. doi:10.1017/S0714980811000304

[5] Wilson, D.M. and Truman, C.D. (2003) Canada’s continuing care population: A description and discussion of information issues and health care concerns. Canadian Journal on Aging, 22, 127-131. doi:10.1017/S0714980800003780

[6] Crotty, M., Miller, M., Whitehead, C., Krishnan, J. and Hearn, T. (2000) Hip fracture treatments - what happens to patients from residential care? Journal of Quality in Clinical Practice, 20, 167-170. doi:10.1046/j.1440-1762.2000.00385.X

[7] Beaupre, L., Cinats, J., Jones, C.A., Scharfenberger, A., Johnston, W.C.D., Senthilselvan, A., et al. (2007) Does functional recovery in elderly hip fracture patients differ between patients admitted from long-term care and the community? Journal of Gerontology Series A-Biological Sciences \& Medical Sciences, 62, 1127-1133.

[8] Beaumpre, L., Jones, C., Majumdar, S., Johnston, D., Buck- ingha, J. and Saunders, L. (2002) Hip fracture in the geriatric population. Alberta Heritage Foundation for Medical Research.

[9] Allen, J., Koziak, A., Buddingh, S., Liang, J., Buckingham, J. and Beaupre, L. (2012) Rehabilitation in patients with dementia following hip fracture: A systematic review. Physiotherapy Canada, 64, 190-201. doi:10.3138/ptc.2011-06BH

[10] Sherrington, C. and Lord, S. (1997) Home exercise to improve strength and walking velocity after hip fracture: A randomized controlled trial. Archives of Physical Medicine and Rehabilitation, 78 208-212. doi:10.1016/S0003-9993(97)90265-3

[11] Stern, P. and Porr, C. (2011) Essentials of accessible grounded theory. Left Coast Press, Inc., Walnut Creek.

[12] Glaser, B. (1965) The constant comparative method of qualitative analysis. Social Problems, 12, 436-445. doi: $10.2307 / 798843$

[13] Streubert Speziale, H. and Rinaldi Carpenter, D. (2007) Qualitative research in nursing: Advancing the humanistic imperative. 4th Edition, Lippincott Williams \& Wilkins, Philadelphia.

[14] Taylor, N., Barelli, C. and Harding, K. (2010) Community ambulation before and after hip fracture: A qualitative analysis. Disability and Rehabilitation, 32, 12811290. doi:10.3109/09638280903483869

[15] Nahm, E., Resnick, B., Orwig, D., Magaziner, J. and DeGrezia, M. (2010) Exploration of informal caregiving following hip fracture. Geriatric Nursing, 31, 254-262. doi:10.1016/j.gerinurse.2010.01.003

[16] Barbour, R. (2007) Doing focus groups. SAGE Publications Ltd., Thousand Oaks.

[17] Friese, S. (2012) Qualitative data analysis with ATLAS.ti. SAGE Publications Ltd., Thousand Oaks.

[18] Beaupre, L.A., Jones, C.A., Johnson, D.W.C., Wilson, D.M. and Majumdar, S.R. (2012) Recovery of function 
following a hip fracture in geriatric ambulatory persons living in nursing homes: REGAIN-I prospective cohort stu- dy. Journal of the American Geriatrics Society, 60, 1268- 1273. doi:10.1111/j.1532-5415.2012.04033.x

[19] Williams, P. (2002) The competent boundary spanner.
Public Administration, 80, 103-124.

http://onlinelibrary.wiley.com/doi/10.1111/1467-9299.002 96/pdf

$\underline{\text { doi: } 10.1111 / 1467-9299.00296}$ 